\title{
Trihexyphenidyl Hydrochloride
}

National Cancer Institute

\section{Source}

National Cancer Institute. Trihexyphenidyl Hydrochloride. NCI Thesaurus. Code C47771.

The hydrochloride salt form of trihexyphenidyl, a synthetic, substituted piperidine with parasympatholytic activity used mostly in Parkinson's disease. Trihexyphenidyl hydrochloride antagonizes activities of acetylcholine via muscarinic receptors. This balances the cholinergic and dopaminergic activity in the basal gang lia leading to an improvement of classic Parkinson symptoms such as rigidity and tremor. In addition, trihexyphenidyl hydrochloride exerts a direct antispasmodic effect on smooth muscle. 